\title{
Análisis de las Prácticas Evaluativas de los Profesores en el Programa a Distancia de Licenciatura en Educación Básica
}

\author{
José E. Cifuentes-Medina \\ Universidad Universidad Pedagógica y Tecnológica de Colombia, Fac. de Estudios a Distancia, Esc. de Ciencias \\ Humanisticas y Educación. Tunja, Colombia (correo-e: joseeriberto.cifuentes@uptc.edu.co)
}

Recibido Mar. 27, 2019; Aceptado May. 25, 2019; Versión final Jul. 5, 2019, Publicado Dic. 2019

\begin{abstract}
Resumen
Se identifica las prácticas evaluativas usadas por los docentes del programa a distancia Licenciatura en Educación Básica y se enmarca en la Educación Superior. El enfoque de investigación es de carácter mixto con apoyo de un cuestionario cerrado. Se indagó acerca de las prácticas de evaluativas que desarrollan los profesores que enseñan en un programa de Licenciatura a Distancia, en la formación de maestros, quienes posiblemente repliquen, en el proceso de enseñanza y aprendizaje, las prácticas de evaluación con las cuales ellos fueron evaluados. Las prácticas evaluativas que se dan en la modalidad a distancia en el programa de Licenciatura en Educación Básica forman parte fundamental del proceso de enseñanza y aprendizaje. Están enmarcadas en el contexto de lo más teórico y no están en concordancia con la esencia del programa que requiere de impulsar más elementos prácticos a través de las actividades evaluables en las sesiones tutoriales.
\end{abstract}

\section{Analysis of the Evaluative Practices of Teachers in the Distance Program Degree of Bachelor in Elementary Education}

\begin{abstract}
The evaluative practices used by teachers of the distance program Bachelor in Basic Education and is part of Higher Education are identified. The research approach is of mixed nature with the support of a closed questionnaire. The questions inquired about the evaluation practices developed by teachers who teach in a Distance Degree program, in the training of teachers, who most probably will replicate, in the teaching and learning process, the evaluation practices that they experienced in their formation. The evaluative practices that are given in the distance modality in the Bachelor in Basic Education program form a fundamental part of the teaching and learning process. They are included in the context of the most theoretical aspects and are not in agreement with the essence of the program that requires to favor more practical elements through the evaluable activities in the tutorial sessions
\end{abstract}




\section{INTRODUCCIÓN}

El presente estudio parte del reconocimiento de que en la actualidad la Universidad es objeto de diversas miradas, estudios criticos y analiticos. En ella se busca por todos los medios su transformación para que sirva como elemento dinamizador de la cultura y de la sociedad que las condiciones de los tiempos actuales reclaman. Es así, que los actores inmersos en el proceso educativo se constituyen en agentes activos de la práctica educativa, en el acto de la enseñanza y del aprendizaje. Esto requiere de docentes y estudiantes comprometidos en el proceso, cuando el estudio se centra en un programa de carácter profesional en la modalidad de educación a distancia tradicional con apoyo de la virtualidad y, en él se busca desentrañar el papel de la evaluación.

En este contexto, es en donde tiene cabida el estudio de una práctica específica que se realiza en la Universidad: la evaluación, la cual se presenta como un elemento nodal en el entramado de la vida escolar, ya que por medio de ella se estima y juzga el valor de lo que se hace en la educación y por lo que hace el docente y el estudiante en particular; es decir, lo que enseñó el maestro y lo que aprendio el estudiante en cada asignatura, se juzga y valora de manera cuantitativa el resultado promedio parcial y final de los conocimientos construidos. Tiene como propósito y objetivo identificar las prácticas evaluativas usadas por los docentes del programa a distancia Licenciatura en Educación Básica y se enmarca dentro de la línea de investigación: Sistemas de evaluación de la calidad educativa. Para el desarrollo de la investigación se tomaron en cuenta aspectos de trabajo que contribuyen a la solidez teórica del proyecto investigativo, a saber: prácticas evaluativas, en la denominación de categoría y subcategorías teóricas, con los respectivos autores. La tabla 1 evidencia el entramado analítico y crítico conlleva a la correlación a debate de cuatro categorías objeto de estudio, en el propósito de la consecución de lograr una meta a través del objetivo general y de la comprobación de la hipótesis de la investigación; en el propósito de una mirada susceptible de análisis para evidenciar la racionalidad de las prácticas evaluativas y su posterior posible transformación en el proceso de enseñanza y aprendizaje para lograr una correlación posible y objetiva de la evaluación.

Tabla 1: Categoría y subcategorías teóricas

\begin{tabular}{|l|l|l|}
\hline \multicolumn{1}{|c|}{ Categoría } & \multicolumn{1}{|c|}{ Subcategoría } & \multicolumn{1}{c|}{ Autores } \\
\hline $\begin{array}{l}\text { La evaluación y las } \\
\text { prácticas evaluativas }\end{array}$ & $\begin{array}{l}\text { Concepto, historia, características, } \\
\text { momentos, ventajas, técnicas, } \\
\text { instrumentos, }\end{array}$ & $\begin{array}{l}\text { Corrales (2008), De la Torre (2009) Ortiz (2015) } \\
\text { Rincón (2010) Moreno (2003) Cajiao y Ortiz (2009) } \\
\text { Santibáñez (2008) }\end{array}$ \\
\hline
\end{tabular}

La evaluación y las prácticas evaluativa: La concepción epistemológica de evaluación y las prácticas evaluativas posibles en Educación a Distancia. Entre los primeros autores se destaca De la Torre (2009) que define la evaluación como "El proceso para determinar hasta qué punto se alcanzan los objetivos o propósitos previamente establecidos del aprendizaje, de una tema o unidad de enseñanza o asignatura o de un nivel educativo; esta es una actividad que forma parte del proceso didáctico y que cobra sentido en el mismo porque permite obtener información respecto a los diversos elementos que confluyen durante la acción pedagógica (De la Torre, 2009)". Ortiz (2015) la evaluación se caracteriza por "ser un proceso sistemático en el que se evidencian dos elementos fundamentales: los objetivos y el contenido de la evaluación. Ambos aspectos están relacionados y son al mismo tiempo los que dan una respuesta correcta a la pregunta: ¿Qué debe ser evaluado?", entre otras cuestiones objeto de análisis en la presente disertación se apoyan en varios auotres.

En tanto, Corrales (2008) sostiene que: la evaluación de los aprendizajes en la educación abierta y a distancia debe fundamentarse en el principio de individualización de la enseñanza; se debe centrar en el alumno y orientarse hacia unos aspectos y propósitos concretos. Estudios coinciden en que la evaluación como De la Torre (2009) que asegura tiene ventajas para el alumno como para el profesor, Ortiz (2015) señala que, es un proceso inherente a la educación superior, que se inserta en el ambiente general de la sociedad... en la que juega un papel fundamental el profesor como guía de este proceso y Corrales (2008) refiere que, la evaluación es un proceso necesario en toda actividad formativa, para conocer y mejorar lo que se hace, mejorar la calidad de formación, conocer el alcance de los objetivos y preparar en calidad de garantía la enseñanza para dinamizar el acto educativo Para avanzar en que la evaluación sea considerada como un proceso según lo afirma Corrales (2008), De la Torre (2009) y Ortiz (2015) se pueden evidenciar tres momentos;

"La evaluación diagnostica permite predecir lo que ocurrirá durante el desarrollo del proceso de enseñanzaaprendizaje o después de él. La evaluación formativa contempla todas las actividades que verifican y controlan el avance logrado, analizando de una manera sistemática los resultados del proceso educativo y la evaluación sumaria es aquella forma de medición y valorización que se utiliza para certificar el aprendizaje, asignar calificaciones, realizar promociones, etcétera (De la Torre, 2009)" 
Autores entre ellos Corrales (2008) coincide con De la Torre (2009) en señalar que "La evaluación en la formación abierta y a distancia no es largo que se realice en un momento determinado, generalmente a final del curso, sino que debe estar presente en diferentes momentos. Desde el inicio con la evaluación diagnostica, a lo largo del curso con la evaluación formativa y al final del proceso de enseñanza aprendizaje con la evaluación sumativa (Corrales, 2008)", contribuyen a generar un análisis y una discusión sustantiva de la evaluación y las práctivas que conllevan a su ejecución connatural de aplicación en el contexto de educación a distancia.

El uso de técnicas e instrumentos para evaluar, está relacionado directamente con la forma de enseñar, es decir que la evaluación tiene un vínculo estrecho con el estilo de enseñanza, Ortiz (2012) señala que "cada docente tiene su propia forma de comunicarse, de planear sus actividades, de utilizar las herramientas que están a su alcance, de evaluar, interactuar con los estudiantes; tolo ello conduce a definirle su propio estilo de enseñanza: por ahora, no es objeto de esta investigación centrarse en los estilos de enseñanza, sería interesante pues la evaluación puede derivar de su modo de enseñanza (Ortiz, 2012)". La mediación entre los estilos de enseñanza y los estilos de aprendizaje está dada por la evaluación, parece contradictorio si es punto de encuentro o convergencia; su llegada es divergente, por la misma relación de los actores, como se evidencia en la figura 1 y que se puede considerar como una proyección del triangulo del acto educativo para lograr la optimización dinámica de interacción de empatia entre los principales actores des tes proceso estudiante y profesor.

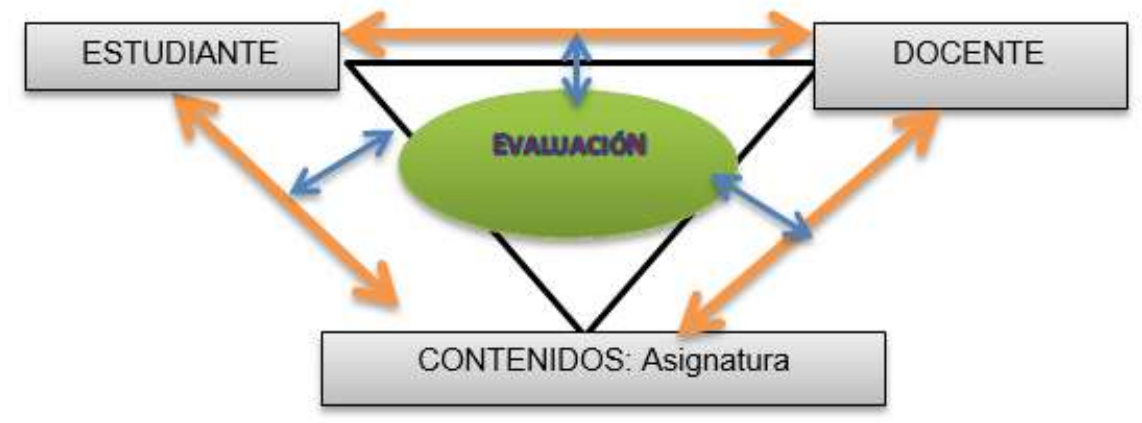

Fig. 1: Triangulo didáctico del acto educativo

El triángulo didáctico del acto educativo como se evidencia en la figura 1 se diseña de acuerdo con De la Torre (2009) y Basabe (2007), es dinámico, sistemático, progresivo, activo; con camino por recorrer para llegar al punto de encuentro, actores del proceso y otros elementos según las necesidades del contexto y el entorno. El triángulo aplica para modalidad de educación a distancia, al igual que para el programa objeto de estudio, en medio de esta realidad para evaluar el aprendizaje los docentes deben recurrir a distintas técnicas y medios (observación directa, interrogatorio verbal, examen práctico, auto informe, prueba, etcétera), e incluso es conveniente que pluralice las fuentes, a fin de juzgar sobre las bases más amplias la eficiencia de la actividad pedagógica. Existen varias técnicas para evaluar en las cuales se utiliza diversos instrumentos como se constata en la figura 2 y, según De la Torre (2009) señala: técnicas informales, técnicas semiformales y técnicas formales. Es preciso que para ahondar en las técnicas e instrumentos que los docentes usan para evaluar el aprendizaje de los estudiantes, la investigación acude a cuatros referentes: Moreno (2003), Corrales (2008), De la Torre (2009) y Rincón (2010). Ninguno de los autores expresa con exactitud cuales técnicas e instrumentos se usan en educación a distancia, son abordadas en términos generales.

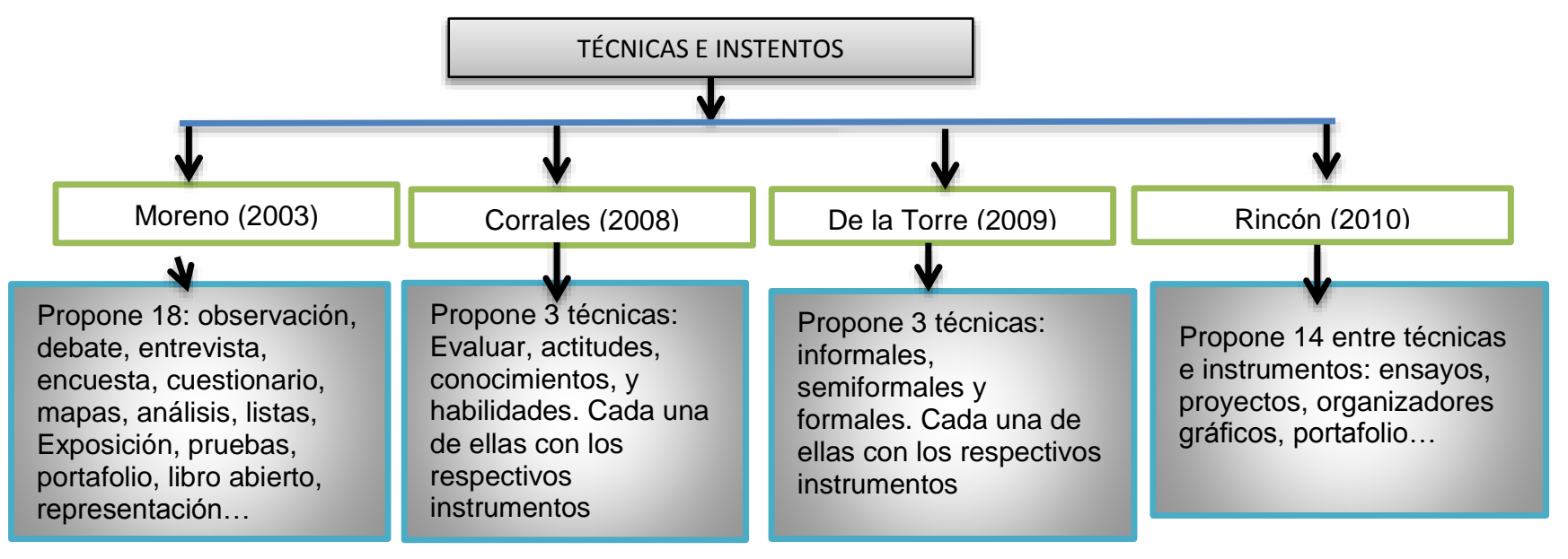

Fig. 2: Aporte de varios autores acerca de las técnicas e instrumentos para evaluar. 
Siempre que se redacten técnicas e instrumentos de evaluación, se deberá utilizar un lenguaje claro, preciso y adecuado al nivel de la población a la que esté dirigida; asimismo, se deberá tener presente que la elaboración adecuada de una evaluación, depende de la creatividad e profesor que la redacta y de su facilidad en el uso del lenguaje. En lo expuesto por Moreno (2003), Corrales (2008), De la Torre (2009) y Rincón (2010) en relación a las técnicas e instrumentos para evaluar los aprendizajes y el proceso de enseñanza, han de guardar coherencia con lo expuesto por Ortiz (2015) quien afirma que, la elaboración de un instrumento de evaluación exige tener en cuenta tres cualidades o requisitos fundamentales: validez, confiablidad y facilidad de empleo. Teniendo en cuenta los tres aspectos coherentes de valido, confiable y objetivas se puede avanzar hacia el éxito del proceso evaluativo como eje mediador del acto educativo. Por otra parte, los instrumentos han de conducir sin otro particular a concebir la evaluación hacia un modelo de evaluación integral y totalizador, por lo tanto, Ortiz (2015) expresa que;

"Ias cualidades de la evaluación en este paradigma integral son: procesual, holística, contextualizada, democrática, formativa: al servicio de los valores y cualitativa. Para dar respuesta a estas exigencias proponemos un modelo evaluativo totalizador, contribuyente a la educación integral del estudiante, y que posee las características siguientes: Evaluación cualitativa, investigativa, continua o del proceso, inicial, de resultados, final, del docente, autoevaluación y coevaluacion (Ortiz, 2015)". Además, de avanzar hacia un nuevo modelo evaluativo integral, totalizador que sea formativo, en donde se pueda conjugar el proceso de evaluación diagnostica, cualitativa, cuantitativa, sumativa, nomotética, ideográfica, inicial, seguimiento y final en un punto único de convergencia y es la evaluación formativa; como lo asegura Ortiz (2015).

En la misma dirección Moreno (2003) señala que: la evaluación formativa o en valores está orientada a la obtención de elementos que, de forma más completa y eficaz, posibiliten la construcción de respuestas morales que sólo son posibles de elaborar en el marco docente-estudiante y viceversa; para fortalecer el proceso de enseñanza y aprendizaje y la interacción en el acto pedagógico, ya que función de la evaluación realimentar las precisiones necesarias para conseguir y lograr aprendizajes significativos y conducir a la construcción de conocimiento. También Arthur W Banger (2004) asegura que: "Tradicionalmente, los cursos basados en el campus se basan en las evaluaciones de los estudiantes para proporcionar a los instructores comentarios sobre su eficacia en la enseñanza. Sin embargo, los instrumentos actuales de evaluación de instructores no aprovechan las prácticas de enseñanza esenciales recomendadas para la enseñanza en línea efectiva". Y se pueden señalar: clase invertida, blended learning, foros, mapas conceptuales, debates, wikis, caza del tesoro, estudio de caso, portafolio, trabajo colaborativo, papa caliente, webquest, blogs y exposiciones virtuales, entre otras estrategias

\section{METODOLOGÍA}

El tipo de investigación aplicado en el estudio fue el descriptivo, según Castillo (2004), por cuanto tiene como propósito describir el objeto estudiado, por ello permite registrar las características, las conductas y demás factores de hechos y fenómenos, busca fundamentalmente describir, pues su objetivo no es comprobar explicaciones, ni hacer predicciones. Con este tipo de investigación se buscó determinar la concepción de evaluación y el uso de las prácticas evaluativas por parte de los docentes en la modalidad a distancia del programa de Licenciatura en Educación Básica.

Hipótesis: Las prácticas evaluativas usadas por los docentes de la Licenciatura en Educación Básica, responden a la emisión de juicios valorativos como resultado de un proceso de enseñanza y aprendizaje pero que están desconectados de la normatividad institucional en cuanto no guradan coherencia y se alejan de la función de la evaluación en el proceso educativo, por el uso repetitivo de las mismas, descuidando procesos como el de Auto evaluación y Coevaluación pero se fortalece la heteroevaluación. Se pretende constatar ¿Cómo se están abordando las prácticas evaluativas usadas por los docentes de educación a distancia del programa Licenciatura en Educación Básica?

Tipo de investigación y Método: El enfoque de la investigación es de carater mixto, como consecuencia de los criterios propios de las ciencias sociales que para la investigación objeto de estudio se aplica los de tipo transversal, en razón a que se aplica a un grupo seleccionado y se recolectan datos en solo momento (Hernández, 2010), buscando describir los datos obtenidos en relación al objetivo de la investigación. El método es deductivo, Ortiz (2012) señala que, va de lo general a lo particular, de lo universal a lo individual; de la teoría a los datos desde una perspectiva mixta.

Población: Para la investigación, la unidad de análisis está representada por los docentes del programa de Licenciatura en Educación Básica, conformada por 40 profesores de tiempo completo, medio tiempo y catedráticos de ambos sexos (24 mujeres y 16 hombres); de un nivel de educación de: pregrado, especialización Maestría y Doctorado, teniendo en cuenta el perfil al cual se han presentado y que orientan 
según sus disciplinas de formación según asignaturas del plan de estudios del programa de Licenciatura en Educación Básica. Programa de pregrado que se ofrece en modalidad a distancia en la Universidad Pedagógica y Tecnológica de Colombia.

Muestra: Para la selección de una muestra aleatoria estratificada se requiere de un marco para cada estrato de población, de los docentes del programa de Licenciatura en Educación Básica. Se trata de un muestreo estratificado, cuando la población no es homogénea, está clasificada según sus características en grupos o estratos y luego al azar se determinan las unidades asegurando la proporcionalidad según los estratos (Niño, 2011). Para lograr determinar el tamaño de la muestra, se acoge a la recomendación técnica, de tomar una muestra equivalente al $30 \%$, es decir, 12 profesores del programa.

\section{RESULTADOS Y DISCUSIÓN}

En esta etapa se contó con la participación de doce docentes, a través de un cuestionario de ocho preguntas cerradas. Hernández Sampieri (2010) señala que un cuestionario, consiste en un conjunto de preguntas respecto de una o más variables a medir, las preguntas cerradas contienen categorías u opciones de respuesta que han sido previamente delimitadas. Las preguntas cerradas del cuestionario con dicotómicas, en el sentido de que los participantes tuvieron la posibilidad de varias posibilidades y opciones de respuesta.

El cuestionario es congruente con el planteamiento del problema e hipótesis que responde a la prácticas evaluativas usadas por los docentes de la Licenciatura en Educación Básica, responden a la emisión de juicios valorativos como resultado de un proceso de enseñanza y aprendizaje y ¿Cómo se están abordando las prácticas evaluativas usadas por los docentes de educación a distancia del programa Licenciatura en Educación Básica? Que luego dio lugar a la sistematización de la información a través de tablas y graficas representativas, acompañadas del análisis consecuente de las mismas.

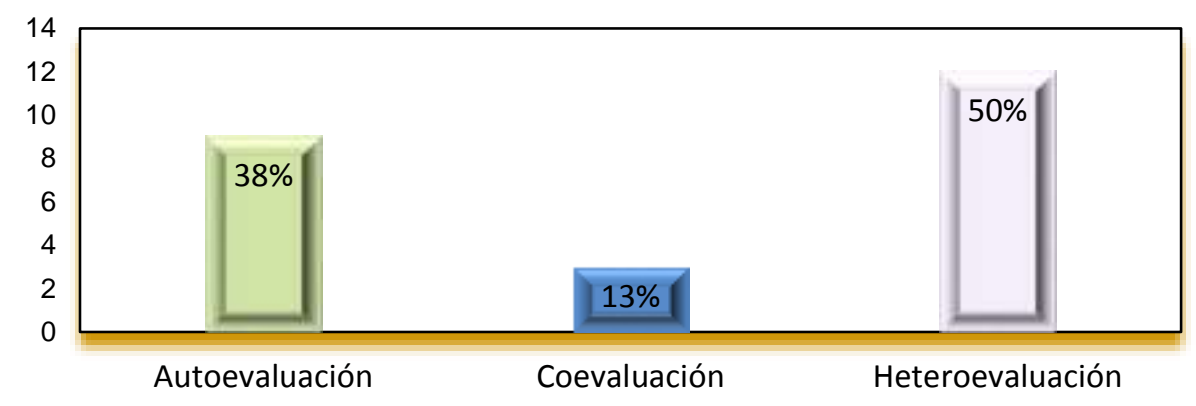

Fig. 3: Procesos de Evaluación

El proceso de evaluación más desarrollado es la heteroevaluaciòn con un $50 \%$ de favoritismo dado permite interactuar entre evaluado y evaluador, el segundo proceso preferido es la autoevaluación con un $38 \%$ dado que no existe la suficiente conciencia de mirarse retrospectivamente a sí mismo, y en último lugar se encuentra la coevaluaciòn con un $13 \%$. Se puede visiblizar en la figura 3, que predomina la heteroevalaucion, es decir, la evaluación que realiza de manera directa profesor a estudiante, dejando del lado la posibilidad de escuchar y leer al estudiante según su autoevaluación y a sus compañeros en la coevaluación, siempre en el respeto y la libertad de cátedra que enmarca la profesión docente.

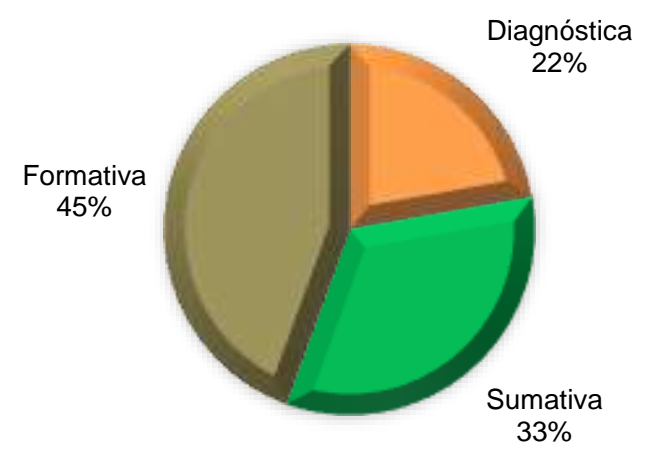

Fig. 4: Momentos de la Evaluación 
El tipo de evaluación preferido por los encuestados corresponde a la formativa con un porcentaje de elección del $45 \%$ ya que es un proceso continuo y constante que permite en gran medida dimensionar al ser humano de manera integral, el segundo tipo elegido corresponde a la evaluación sumativa con un porcentaje del $33 \%$ manifestando que es un proceso arduo que implica mayor dedicación para evidenciar el avance de los aprendizajes y en menor preferencia se encuentra con un $22 \%$ el tipo de evaluación diagnóstica, dado que lo usan para recibir o empalmar con los grupos al comienzo de las asignaturas especialmente. Es consonancia con el visible en la figura 4, conlleva a propender por la necesaria correlación entre los tres momentos necesarios de la evaluación desde el punto de partida de los presaberes o inicio del proceso, se decir, el antes pero también el durante y la salida del proceso de enseñanza y aprendizaje para establecer una correlación de análisis y realimenación constructiva y significativa.

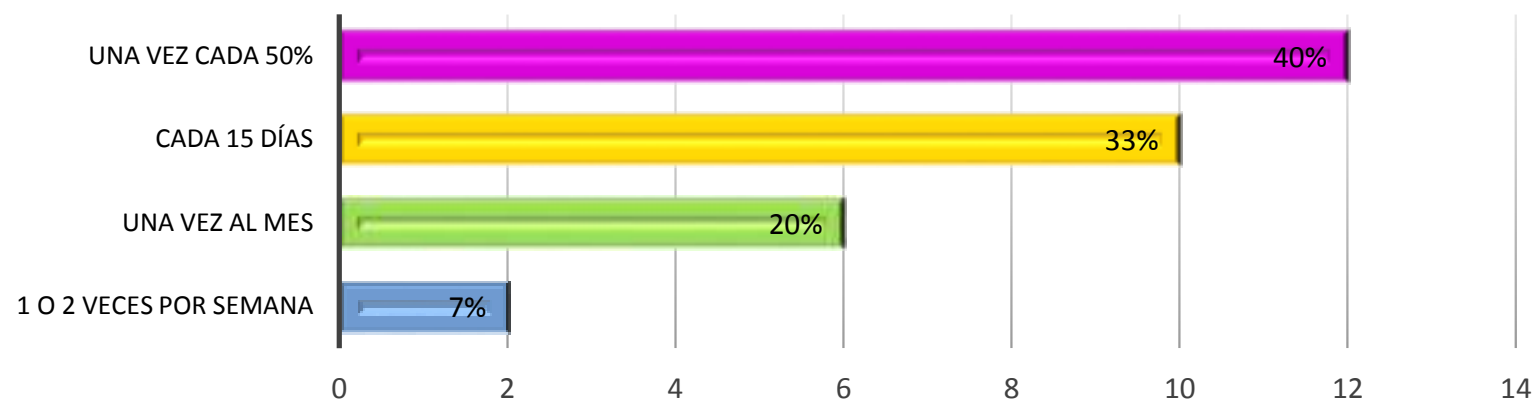

Fig. 5. Periodicidad de la evaluación

El $60 \%$ de los encuestados manifiesta realizar evaluaciones cotidianamente entre 1 o 2 veces por semana, 1 vez al mes, o cada 15 días lo que mostraría que hacen seguimiento al avance de aprendizajes y acompañamiento a los estudiantes, mientras que el $40 \%$ restante dice evaluar 1 vez para sacar la evaluación definitiva. En la figura 5, se evidencia el papel que ocupa el corte de semestre, es decir, la división del mismo para efectos de sistematización de evalución entendida esta quizás como un test de salida a un proceso de enseñanza y aprendizaje de los contenidos de una asignaura en cada mitad de semestre.

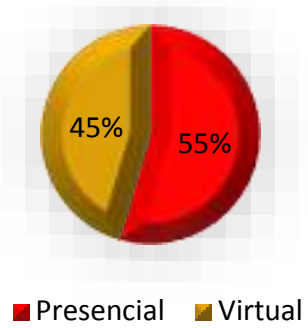

Fig. 6. Encuentros en donde realiza la evaluación

El $55 \%$ de las personas manifiesta preferencia a la hora de realizar evaluaciones en los encuentros presenciales ya que le permite una mayor evidencia, transparencia y asertividad en el proceso por el hecho de la observación, mientras que el restante $45 \%$ presenta cierto agrado por la realización de evaluaciones de manera virtual dado que permite la aceleración de resultados desde la plataforma por su programación efectiva. Se considera que se continua refieriendo por parte de los profsores a la aplicación de los test o pruebas especificas de selección multiple y demás en el sentido de examen pero se puede inferir que no se aborda el proceso de evaluación de manera integral, objetiva y sustantiva en el acto educativo de profesor y estudiante según la disciplina o asignatira que establece o conlleva a esta connotación de relación humana y por la construcción de conocimiento.

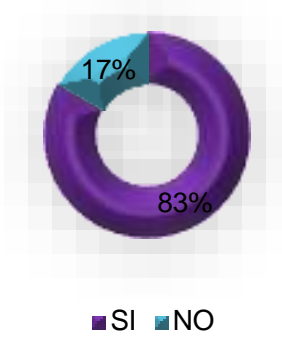

Fig. 7. Rubricas de evaluación 
El $83 \%$ de los docentes encuestados manifiestan que usan las rúbricas para la evaluación de actividades ya que éstas le permiten al estudiante una mayor retroalimentación de talleres, actividades en sí, sin embargo, el restante $17 \%$ dice no usar las rúbricas porque a la hora de digitarlas en plataforma es un proceso dispendioso que lleva bastante tiempo y debe ser corregida muy bien antes de presentarla a sus estudiantes. Se evidencia a través de los resultadod de la figura 7, que los profesores realizan la evaluación de las actividades de los estudiantes a través de rubricas diseñadas quizás según la disciplina o área de enseñanza y el tipo de actividad objeto de evaluación sistematica que a la par ha de ser progresiva.

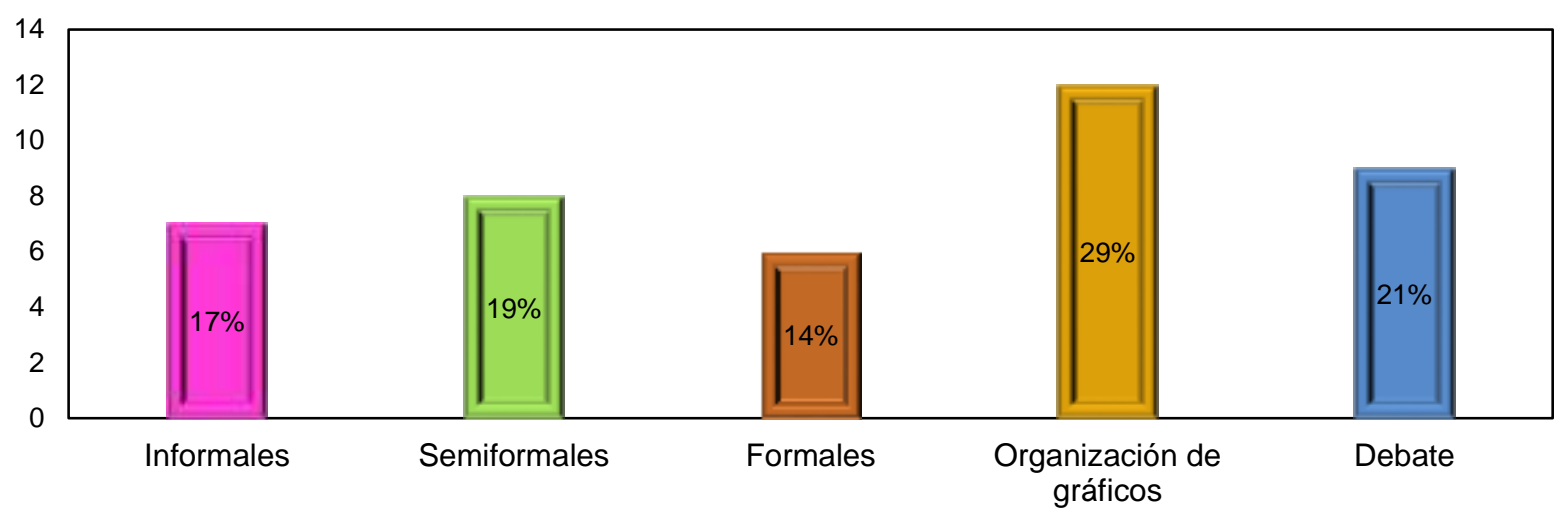

Fig. 8. Técnicas de evaluación

La técnica de evaluación más utilizada es la organización de gráficos con un 29\% de favorabilidad entre los encuestados, ya que argumentan que la parte visual es muy llamativa para la educación a distancia y virtual, además es un método que condensa cualquier cantidad de información desde lecturas cortas o amplias, a esta técnica le siguen, el debate con un $21 \%$ ya que este permite conocer el argumento de opinión de los estudiantes frente a los diverso temas, las semiformales con un 19\% y con menor preferencia las formales con un $14 \%$. En la figura 7 se puede constatar que lo mas usado por los docentes como quizás exigencia de probación de los aprendizajes de temas, contenidos establecidos o estandarizados en una asignatura se dinamicen, socialicen o se realicen a través de organizadores graficos y su respectiva connotación objetiva de implicación en el proceso de evaluación de los aprendizajes y de la realimentación que pueda surgir desde la enseñanza luego si hay convicción de esta relación y del papel que los estudiantes ocupan en el acto educativo.

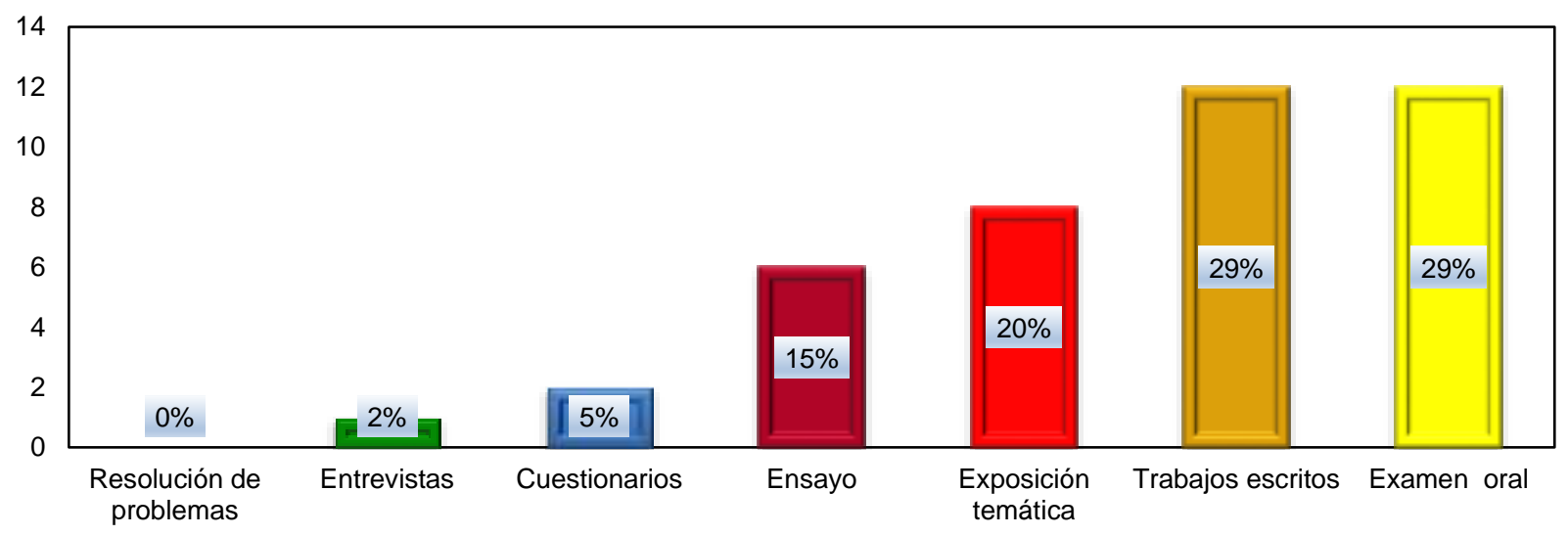

Fig. 9. Instrumentos de evaluación

Los instrumentos de evaluación menos usados son la resolución de problemas con un $0 \%$ y las entrevistas con un $2 \%$, debido a que requieren un mayor pensamiento crítico a la hora de abordar a los estudiantes, y desde el medio virtual por ejemplo, es difícil lograr una entrevista de manera sincrónica, con un $5 \%$ se encuentran los cuestionarios no permiten argumentación o justificación, los docentes dicen que para ellos es un instrumento cerrado, el ensayo con un $15 \%$ ya que en variadas ocasiones se presenta fraude, es decir, copia de texto de otros autores, no se respeta la propiedad intelectual especialmente frente a los contenidos de internet, la exposición temática con un $20 \%$ cuando el encuentro presencial lo permite, trabajo escrito y examen oral con un $29 \%$ dan cuenta del conocimiento, fluidez verbal, capacidad de argumentación, síntesis, procesos. 


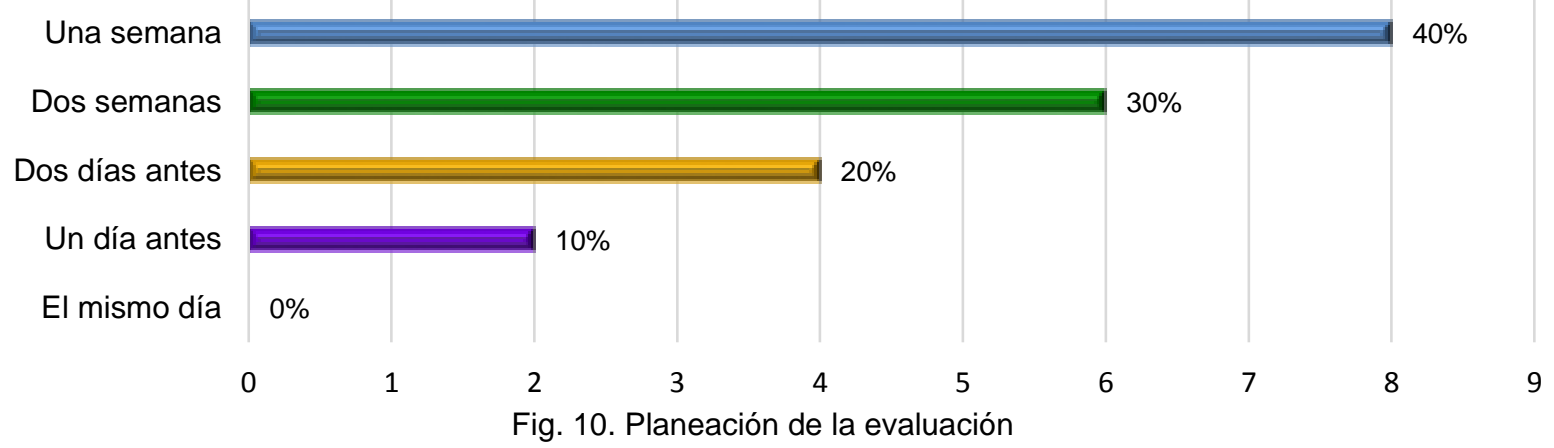

Por profesionalismo o ética los docentes encuestados manifiestan no planear la evaluación el mismo día de la aplicación, aunque un 10\% dice planearla el di anterior pues les permite según ellos recoger todos los temas vistos, un $20 \%$ dos días antes por organización de actividades para ellos, el $30 \%$ dice dos semanas para indicar que se ajuste a los requerimientos del tema y un $40 \%$ manifiesta que requiere de una semana de forma que pueda ajustarse a los intereses de los estudiantes y requerimientos de la asignatura.

\section{CONCLUSIONES}

De acuerdo al trabajo presentado, a las discusiones sobre las diversas propiedades estudiadas, y a los resultados obtenidos en cada caso, se pueden plantear las siguientes conclusiones principales:

1. La evaluación se constituye en un principio del acto educativo, es el punto de convergencia y encuentro entre el arte de enseñar y la convicción de aprender; pues los actores han de buscar la construcción de conocimiento y, la comprensión de la realidad que se constituye en el logro de un trabajo mancomunado entre docente y estudiante.

2. La investigación acerca de las prácticas evaluativas en la modalidad a distancia en el programa de Licenciatura en Educación Básica se ajustó al contexto de los docentes de las diferentes áreas del conocimiento según el plan de estudios y estudiantes de diferentes semestres, logrando así la participación de los actores del proceso de enseñanza y aprendizaje, en donde se gesta la evaluación y hace parte de la vida diaria de docentes y estudiantes.

3. La flexibilidad de la educación a distancia con el apoyo de la plataforma virtual permite planear y organizar el proceso educativo y acto de la evaluación que favorece a los docentes y estudiantes, implicando el cambio de perspectivas enfocadas a la construcción de conocimiento en cada una de las asignaturas del programa de Licenciatura en Educación Básica.

4. En el proceso de evaluación se puede constatar que el $50 \%$ se apoya de la heteroevaluación de manera directa de profesor a estudiante sin lugar a autoevaluación y/o coevalación. Se puede demarcar que que el proceso de la evalucipon ocupa un lugar la formativa y es de aclarar que la diagnostica es valida por cuant, no se ha desconocer los presaberes o saberes con los cuales el estudiante ya presenta cierto nivel de conocimiento.

5. Los trabjos escritos y los exámenes orales ocupan el mismo porcentaje entre los más usados en el proceso de evaluación y entre las técnicas la mas usada corresponde a los organziadores graficos como solicitud expresa de representar de manera visual los saberes de los contenidos temáticos de las asiganturas que orientan los docentes.

\section{AGRADECIMIENTOS}

El autor agradece el apoyo de la Dra. Manuela del Carmen Sánchez Humaran profesora de la Universidad de Baja California -México, por el acompañamiento en la Estancia de Investigación y producción Científica.

\section{REFERENCIAS}

Banger, A.W., The Seven Principles of Good Practice: A framework for evaluating on-line teaching programs, The internet and higher Education, 7:3, 217-232, doi.org/10.1016/j.iheduc.2004.06.003 (2004)

Basabe, F. Educación a distancia en el nivel superior. 1a ed. México: Editorial Trillas. (2007). 
Cajiao, F. (2.009). Pertinencia y Evaluación, dos claves para mejorar la calidad. En Memorias II Foros y experiencias significativas 2.009, Tunja, Octubre de 2.009. Secretaria de Educación de Boyacá.

Castillo, S. M. Guía para la formulación de proyectos de investigación. 1aㅡ ed. Bogotá: Ed. Magisterio. (2004)

Corrales, M. I. Metodología de la formación abierta y a distancia. 1aㅡ ed. Balderas México: Ed. Limusa. (2008)

De la Torre, Z. F. 12 Lecciones de pedagogía, educación y didáctica. 1ª Ed. México: Ed. Alfaomega. (2009)

Hernández, S. R. et I Metodología de la investigación. 5a ed. Colombia: Editorial McGraw W-ILL (2010).

Lerma, H. Metodología de la investigación: Propuesta, anteproyecto y proyecto. 4ª ed. Bogotá: Ecoe (2009)

Moreno, H. Técnicas de evaluación. 1aㅡ ed. Bogotá: Editorial Magisterio. (2003).

Niño, R. V. M. Metodología de la investigación. 1aㅡ ed. Bogotá: ediciones de la U. (2011)

Osorio, V. M. "Las prácticas evaluativas. Una reflexión necesaria en el contexto de la educación superior". Revista Investigación Bolivariana, Barranquilla, 2: 149 - 154. (1999).

Ortiz, F. J. Políticas y experiencias de evaluación en la Educación Superior en Colombia 1aㅡ ed. Observatorio Nacional de Políticas en Evaluación Educativa. Impreso en Colombia. (2015).

Ortiz, F. Metodología de la investigación: el proceso y sus técnicas. 1aㅡ ed. México: Ed. Trillas (2012).

Rincón, E. La evaluación y la evaluación por competencias. Seminario Permanente de pedagogía. 1a ed. Tunja: Imprenta y publicaciones UPTC. (2010)

Jo Ann y R. Coe y E. Doreen. An Evaluation of Teaching Direct Practice Courses in a Distance Education Program for Rural Settings, Journal of Social Work Education, 35:3, 353-365, DOI: 10.1080/10437797.1999.10778974 (1999)

Gibbs, G., y M. Coffey, The Impact Of Training Of University Teachers on their Teaching Skills, their Approach to Teaching and the Approach to Learning of their Students. Active Learning in Higher Education, 5(1), 87-100. https://doi.org/10.1177/1469787404040463 (2004). 
